\title{
ANALYTIC MODFET MODELS BEYOND THE TRIANGULAR WELL APPROXIMATION
}

\author{
Timothy J. Drummond and Marc E. Sherwin \\ Center for High Frequency Microelectronics, Solid State Electronics Laboratory, Department of Electrical \\ Engineering and Computer Science, University of Michigan, Ann Arbor, MI 48109-2122, U.S.A.
}

(Received 7 September 1989; in revised form 6 December 1989)

\begin{abstract}
A new approach to modeling normal interface modulation doped field-effect transistors (MODFETs) is presented in which the 2-D electron gas density is integrated along the channel instead of the channel potential. With this approach we are able to implement a highly accurate polynomial description of the Fermi potential in the channel as a function of free electron density for arbitrary acceptor densities in GaAs channel. A substantial increase in the near threshold device current results. A carrier density dependent low field mobility is also incorporated into the model. These modifications are expected to result in an improved ability to estimate device characteristics from extrinsic material and device parameters.
\end{abstract}

$n_{\mathrm{s}} \quad$ the sheet electron density in the channel $\left(\mathrm{m}^{-2}\right)$ $n_{\mathrm{s} 0} \quad$ the maximum sheet electron density in the channel $\left(\mathrm{m}^{-2}\right)$

$N \quad$ a polynomial function of $n_{\mathrm{s}}\left(\mathrm{Vm}^{-2}\right)$ $\left(\mathrm{eVm}^{2}\right)$ and $V_{\mathrm{Fi}}\left(n_{\mathrm{s}}\right)\left(\mathrm{Vm}^{2}\right)$ the channel $(\mathrm{m})$

the doped AlGaAs thickness beneath the gate (m) the undoped AlGaAs spacer layer thickness at the heterojunction $(\mathrm{m})$

a derived constant (m)

a two dimensional density of states in the channel $\left(\mathrm{V}^{-1} \mathrm{~m}^{-2}\right)$ quantum well (J)

a constant in the linear approximation of $E_{\mathrm{Fi}}\left(n_{\mathrm{s}}\right)$ (J)

the AlGaAs/GaAs heterojunction discontinuity (J) the static permittivity of the AIGaAs layer $\left(\mathrm{Fm}^{-1}\right)$

the electric field in the channel $\left(\mathrm{Vm}^{-1}\right)$ the critical electric field at the onset of electron velocity saturation in the channel $\left(\mathrm{Vm}^{-1}\right)$

a constant in the cubic approximation of $V_{\mathrm{Fi}}\left(n_{\mathrm{s}}^{1 / 3}\right)$ $\left(\mathrm{Vm}^{4 / 3}\right)$

the gate metal work function (V)

a constant exponent of $n_{\mathrm{s}}$ describing the carrier density dependence of mobility

scale factors relating the potentials of the ground and lst excited states in the quantum well to $n_{s}^{2 / 3}$ $\left(\mathrm{Vm}^{4 / 3}\right)$

the reduced Plank's constant (Js)

the transistor drain current (A)

the saturated transistor drain current (A)

Boltzmann's constant $(\mathrm{J} / \mathrm{K})$

the transistor gate length $(\mathrm{m})$

the effective mass of an electron in the channel (kg)

the mobility of an electron in the channel $\left(\mathrm{m}^{2} \mathrm{~V}^{-1} \mathrm{~s}^{-1}\right)$

the mobility of an electron in the channel for the maximum carrier density in the channel $\left(\mathrm{m}^{2} \mathrm{~V}^{-1} \mathrm{~s}^{-1}\right)$
$N_{\mathrm{d}} \quad$ the donor doping level in the AlGaAs $\left(\mathrm{m}^{-3}\right)$

a constant in the linear approximation of $E_{\mathrm{Fi}}\left(n_{\mathrm{s}}\right)$

the total AlGaAs thickness between the gate and

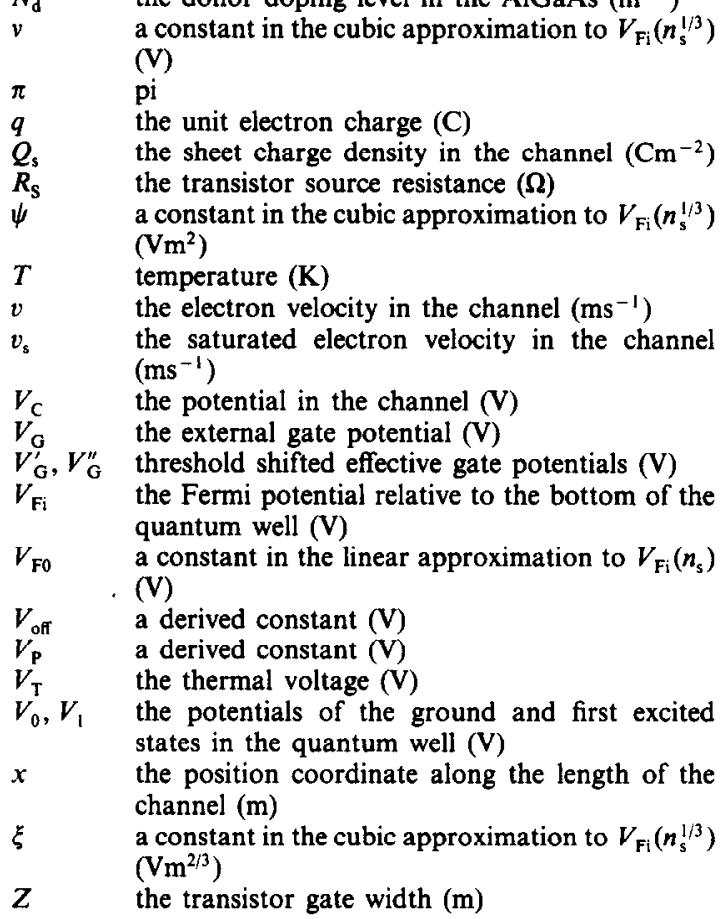

The original device model for the normal interface modulation-doped field-effect transistor assumed a two-piece linear velocity-field characteristic in an infinite triangular quantum well[1]. To obtain a solution within the gradual channel approximation, the Fermi energy in the channel was held to be a constant. The first improvement to be offered to this model was to incorporate a linearized Fermi energy versus channel carrier density of the form $E_{\mathrm{F}}=a n_{\mathrm{s}}+E_{\mathrm{F} 0}[2]$. Although it is not widely recognized, this improvement was presented simultaneously by Delagebeaudeuf $e t$ al.[3] where they also noted that the low field mobility is apparently a 
power law function of the two-dimensional electron gas density of the form $\mu=\mu_{0} n_{\mathrm{s}}$. This relation agrees with the original observation of Tsui et al.[4] and the subsequent work of Hirakawa and Sasaki[5]. This observation has been largely neglected in subsequent modeling papers. Instead, work has focussed on the implementation of various velocity-field descriptions[6] and the description of the current-voltage characteristics past the onset of saturation[6-9]. This paper focusses instead upon the implementation of an improved description of the Fermi energy in the channel and a carrier density dependent low field mobility.

In deriving the $I-V$ characteristics of any fieldeffect transistor, the first step is the derivation of the charge control equation relating the gate potential to the channel carrier density for zero drain bias. The standard charge control equation for a modulation doped structure is,

$Q_{\mathrm{s}}=q n_{\mathrm{s}}=(\epsilon / d)\left(V_{\mathrm{G}}-E_{\mathrm{Fi}} / q-\phi_{\mathrm{m}}+V_{\mathrm{P}}+\Delta E_{\mathrm{c}} / q\right)$

where $Q_{\mathrm{s}}$ is the areal charge density in the channel, $q$ is the electronic charge, $\epsilon$ is the permittivity of the AlGaAs layer, $d$ is the thickness of the AlGaAs layer separating the gate electrode from the channel, $V_{\mathrm{G}}$ is the gate bias, $E_{\mathrm{Fi}}$ is the Fermi energy in triangular quantum well relative to the bottom of the well, $\phi_{\mathrm{m}}$ is the metal/AlGaAs Schottky barrier height, $V_{\mathrm{P}}=q N_{\mathrm{d}} d_{\mathrm{d}}^{2} / 2 \epsilon$ and $\Delta E_{\mathrm{c}}$ is the conduction band offset at the $\mathrm{AlGaAs} / \mathrm{GaAs}$ heterojunction. In defining the constant $V_{\mathrm{P}}, N_{\mathrm{d}}$ is the donor doping level in the AlGaAs, $d_{\mathrm{d}}$ is the thickness of the doped AlGaAs. The total AlGaAs thickness is assumed to consist of two regions, a doped region immediately below the gate and a thin undoped spacer layer at the heterojunction of thickness $d_{\mathrm{s}}$ so that $d=d_{\mathrm{d}}+d_{\mathrm{s}}$. To obtain notational consistency the energy terms in eqn (1) are redefined as $V_{\mathrm{Fi}}=-E_{\mathrm{Fi}} / q$ and $\Delta V_{\mathrm{c}}=-\Delta E_{\mathrm{c}} / q$. The notation is further simplified by defining

$$
V_{\text {off }}=\phi_{\mathrm{m}}-V_{\mathrm{p}}+\Delta V_{\mathrm{c}}
$$

and

$$
V_{\mathrm{G}}^{\prime}=V_{\mathrm{G}}-V_{\text {off }}
$$

so that eqn (1) may be rewritten as

$$
Q_{\mathrm{s}}=q n_{\mathrm{s}}=(\epsilon / d)\left(V_{\mathrm{G}}^{\prime}+V_{\mathrm{Fi}}\right) .
$$

Continuing the derivation of the MODFET $I-V$ characteristics Schockley's gradual channel approximation is assumed to be valid. In this case one writes the drain current as $I_{\mathrm{D}}=Z v(x) Q_{\mathrm{s}}(x)$ with $Q_{\mathrm{s}}$ modified to

$$
Q_{s}(x)=(\epsilon / d)\left[V_{\mathrm{G}}^{\prime}+V_{\mathrm{Fi}}(x)-V_{\mathrm{C}}(\mathrm{x})\right]
$$

where $V_{c}(x)$ is the voltage drop along the length of the channel beneath the gate. Analytic solution of the $I-V$ characteristics requires two further substitutions. Simple representations must be obtained for both $v(x)$ and $V_{\mathrm{Fi}}(x)$. The simplest approximation for $v(x)$ is the two piece linear model for which $v(x)=\mu F(x)=\mu\left(\mathrm{d} V_{\mathrm{C}} / \mathrm{d} x\right)$ where $F(x)$ is the electric field in the channel for fields less than a critical field defined as $F_{\mathrm{c}}=v_{\mathrm{s}} / \mu$ where $v_{\mathrm{s}}$ is an effective saturation velocity. For fields larger than $F_{\mathrm{c}}$, $v(x)=v_{\mathrm{s}}$. A drawback of this model is that the transition from triode operation to saturation is not smooth. To circumvent this is number of alternative velocity-field characteristic forms have been investigated.

One approximation which offers a smooth transition to saturation while preserving a simple, explicit analytic expression for $I_{\mathrm{D}}$ is

$$
v(x)=\mu F(x) /\left[1+\left(\mu / v_{\mathrm{s}}\right) F(x)\right] .
$$

Equation (6) has been implemented for $\mu$ constant[10] and $\mu$ proportional to $n_{\mathrm{s}}^{\gamma}$ where $\gamma$ is an exponent in the range $0.3-2[11]$. The resulting drain $I-V$ characteristics for these and other approximations which are not necessarily analytic are more esthetically pleasing than the two-piece linear approximation but it is not clear that they provide an improved description of the device constants which must be adjusted to fit experimental device characteristics. Of the various approximations to the electron velocity-field characteristics eqn (6) represents the most gradual approach to saturation while the twopiece linear approximation describes the most rapid. Consequently, analysis of these two representations should most clearly illustrate the impact that the choice of a particular velocity-field curve may have on the resulting current transfer characteristics.

Rather than trying to justify a particular velocityfield characteristic, the central issue of this discussion is to demonstrate an improved representation for $V_{\mathrm{Fi}}(x)$. Within the triangular well approximation, the sheet carrier density for two energy levels in the well is given by

$$
\begin{aligned}
& n_{\mathrm{s}}=D V_{\mathrm{T}} \ln \{1+\exp {\left[\left(V_{\mathrm{Fi}}-V_{0}\right) / V_{\mathrm{T}}\right] } \\
& \times\left(1+\exp \left[\left(V_{\mathrm{Fi}}-V_{1}\right) / V_{\mathrm{T}}\right]\right\}
\end{aligned}
$$

where $D$ is the two dimensional density of states, $V_{\mathrm{T}}$ is the thermal voltage $(k T / q)$ and $V_{0}$ and $V_{1}$ are the potentials of the ground and first excited state in the triangular quantum well. The ground and excited state potential can be approximated as $V_{0}=-\gamma_{0} n_{\mathrm{s}}^{2 / 3}$ and $V_{1}=-\gamma_{1} n_{\mathrm{s}}^{2 / 3}$ and $D=q m^{*} / \pi \hbar^{2}$. The constants $D, \gamma_{0}$ and $\gamma_{1}$ have been adjusted slightly to obtain agreement with experimental data[1]. Substituting for $V_{0}$ and $V_{1}$ in eqn (7) one finds that it is implicit in $n_{5}$ and that an explicit and integrable approximation must be substituted. In the seminal analysis[1], $V_{\mathrm{F}}$ was simply assumed to be a small quantity and was set equal to zero. In subsequent refinement the relation $V_{\mathrm{Fi}}\left(n_{\mathrm{s}}\right)$ was linearized at a carrier density of approximately $8 \times 10^{11} \mathrm{~cm}^{-2}$, close to the upper limit for 2-D equilibrium electron densities commonly encountered in AlGaAs/GaAs hetcrojunctions[2,3].

Linearizing eqn (7) yielded an expression of the form $V_{\mathrm{Fi}}=V_{\mathrm{Fo}}-a n_{\mathrm{s}}$. Substituting this into eqn (5) 
leads to

$$
Q_{\mathrm{s}}=[\epsilon / q(d+\Delta d)]\left[V_{\mathrm{G}}^{\prime}+V_{\mathrm{F} 0}-V_{\mathrm{C}}(x)\right]
$$

where $V_{\mathrm{F} 0}$ is a function of $T$ and $\Delta d=\epsilon a / q \simeq 8 \mathrm{~nm}$. In this approximation, $V_{\mathrm{F} 0}$ is grouped with the terms composing $V_{\text {off }}$. For clarity in later discussion a new term $V_{\mathrm{G}}^{\prime \prime}=V_{\mathrm{G}}-V_{\text {of }}+V_{\mathrm{F} 0}$ is defined. Equation (8) then allows us to write the drain current as

$$
I_{\mathrm{D}}=Z \mu[\epsilon / q(d+\Delta d)]\left[V_{\mathrm{G}}^{\prime \prime}-V_{\mathrm{C}}(x)\right]\left(\mathrm{d} V_{\mathrm{C}} / \mathrm{d} x\right)
$$

which is easily separated into integrals over $x$ and $V_{C}$ assuming $I_{\mathrm{D}}$ constant along the channel. The limits of integration are defined by the physical distance along the length of the gate, $x^{\prime}=0$ to $x$ and the integration over $V_{\mathrm{C}}$ proceeds from $V_{\mathrm{C}}\left(x^{\prime}=0\right)=R_{\mathrm{S}} I_{\mathrm{D}}$ with $R_{\mathrm{S}}$ being the source resistance of the transistor, to $V_{\mathrm{C}}\left(x^{\prime}=x\right)$. Solving explicitly for $V_{\mathrm{C}}(x)$ and taking the derivative with respect to $x$ results in an expression for $F(x)$ which when equated to $F_{\mathrm{c}}$ at $x=L$ ( $L=$ the length of the gate) defines the saturation drain current, $I_{\mathrm{D} \text {, sat }}$. The resulting equation for $I_{\mathrm{D} \text {, sat }}$ is a compact function of the maximum carrier density, the saturation velocity and the source resistance.

Beyond linearizing eqn (7) few attempts have been made to obtain an improved description of the carrier density along the channel. Khondker et al. proposed a gate voltage dependent $\Delta d$ in eqn (8) and this suggestion has been implemented by Hughes and Snowden[12,8]. This, however, represents an incomplete approximation. Although $\Delta d$ has assumed an independent identity as representing the average distance of an electron in the GaAs from the hetrojunction it is, in fact, obtained directly from algebraic operations leading to eqn (8) and is directly proportional to the slope of eqn (7) at the point of linearization. The linearization process also yields the zero intercept $V_{\mathrm{ro} 0}$. Consequently, if $\Delta d$ is to be made a function of $V_{\mathrm{G}}$ then so must $V_{\mathrm{F} 0}$. From eqn (7) analytic expressions can be derived for $V_{\mathrm{F} 0}\left(n_{\mathrm{s}}\right)$ thereby making $\Delta d$ and $V_{\mathrm{G}}^{\prime \prime}$ explicit function of $n_{\mathrm{s}}$. For purposes of later comparison this approach, referred to herein as the multi-linear approximation (as opposed to the original single-linear model), has been implemented for comparison with the more accurate model to be derived. In more original approaches a purely empirical expression for $n_{\mathrm{s}}$ has been suggested by Rhodin and Roblin[9] but is integrable only for the intrinsic FET with $R_{\mathrm{S}}=0$. Kola et al. have proposed writing $V_{\mathrm{Fi}}$ as a quadratic function of $n_{\mathrm{s}}$ although the improvement over the linear approximation is questionable and its implementation in a device model has not apparently been published[13].

Motivation for the present analysis follows from the fact that when the drain current is derived as an integral over $V_{C}(x)$ the most sophisticated expression for $V_{\mathrm{Fi}_{\mathrm{i}}}$ which allows analytic integration of an expression similar to eqn (9) is of the form $V_{\mathrm{Fi}}=V_{\mathrm{F} 0}-a n_{\mathrm{s}}^{\gamma}$ where $\gamma$ is and arbitrary exponent. By writing the right hand side of eqn (9) as an integral over $n_{\mathrm{s}}$ much more sophisticated representations of $V_{\mathrm{Fi}}\left(n_{\mathrm{s}}\right)$ are allowed. Toward the end of selecting a simple more accurate representation it is recognized that an intelligent choice of basis functions to represent $V_{\mathrm{Fi}}$ should result in a minimum number of fitting coefficients to obtain a smooth and accurate approximation. Moloney et al. have proposed

$$
E_{\mathrm{Fi}}(\mathrm{meV})=234.5\left(n_{\mathrm{s}} / 10^{12} \mathrm{~cm}^{-2}\right)^{1 / 3}-155
$$

and

$$
E_{\mathrm{Fi}}(\mathrm{meV})=97.6\left(n_{\mathrm{s}} / 10^{12} \mathrm{~cm}^{-2}\right)^{1 / 3}(@ 77 \mathrm{~K})
$$

to be useful in the modeling of the gate capacitance-voltage characteristics of MODFETs[14]. Justification for this particular functional form has been discussed by Shey and $\mathrm{Ku}[15]$.

This logic is extended to show that an extremely accurate description of $V_{\mathrm{Fi}}$ (in the triangular well approximation) may be obtained by expanding (7) as a polynomial function of $n_{s}^{1 / 3}$ rather than $n_{s}$ as proposed by Kola et al. An appropriate range of sheet carrier densities for AlGaAs/GaAs heterojunctions is $2 \times 10^{10}$ to $2 \times 10^{12} \mathrm{~cm}^{-2}$. For this range of $n_{\mathrm{s}}$ we investigated the least squares fitting of polynomials in $n_{\mathrm{s}}^{1 / 3}$ to eqn (7) up to third order with various coefficients forced to zero. This led to the selection of a full cubic formula

$$
V_{\mathrm{Fi}}=\psi n_{\mathrm{s}}+\phi n_{\mathrm{s}}^{2 / 3}+\xi n_{\mathrm{s}}^{1 / 3}+v
$$

at both 300 and $77 \mathrm{~K}$. The coefficients thus determined are given in Table 1. Figure 1 compares the linear approximation, the quadratic approximation of Kola et al. [13], the approximation of Shey and $\mathrm{Ku}$ $\left(E_{\mathrm{F}}=E_{\mathrm{F} 0}+a n_{\mathrm{s}}^{2 / 3}\right)[15]$ and eqn (12) at room temperature. In the scale of the figure the cubic fit in $n_{\mathrm{s}}^{1 / 3}$ is indistinguishable from eqn (7). Alternatively, we show the error $\left|\left(V_{\mathrm{Fi}}^{*}-V_{\mathrm{Fi}}\right) / V_{\mathrm{Fi}}\right|$ in the top half of the figure where $V_{\mathrm{Fi}}^{*}$ represents the various approximate functions excepting the linear approximation (not shown). Equation (10) is not shown because it is not derived from a fit within the triangular well approximation and also assumes a finite acceptor density $\left(3 \times 10^{14} / \mathrm{cm}^{3}\right)$ in the GaAs.

Going beyond the triangular well approximation, eqns (10) and (11) illustrate the feasibility of fitting the results of a self consistent solution of Poisson's and Schroedinger's equation for a modulation doped quantum well. Moloney et al. claim the analytic functions are accurate to within $1 \%$ in the range $1-10 \times 10^{11} / \mathrm{cm}^{2}$. Application of the functions is

Table 1 . Coefficients of the polynomial representation of $V_{\mathrm{Fi}}$ in the triangular quantum well (TQW) at two temperatures as a cubic function of $n_{s}^{1 / 3}$

\begin{tabular}{ccccc}
\hline$T$ & $\psi$ & $\phi$ & $\xi$ & $v$ \\
\hline $300 \mathrm{~K}$ & -0.09332812 & 0.14693080 & -0.3742477 & 0.1948085 \\
$77 \mathrm{~K}$ & -0.05099231 & -0.03042406 & -0.1053764 & 0.04162516 \\
\hline
\end{tabular}

The coefficients assume that $n_{\mathrm{s}}$ is in units of $10^{12} \mathrm{~cm}^{-2}$. An equivalent representation of $E_{\mathrm{Fi}}$ in $\mathrm{eV}$ is obtained by reversing the sign of each coefficient. 

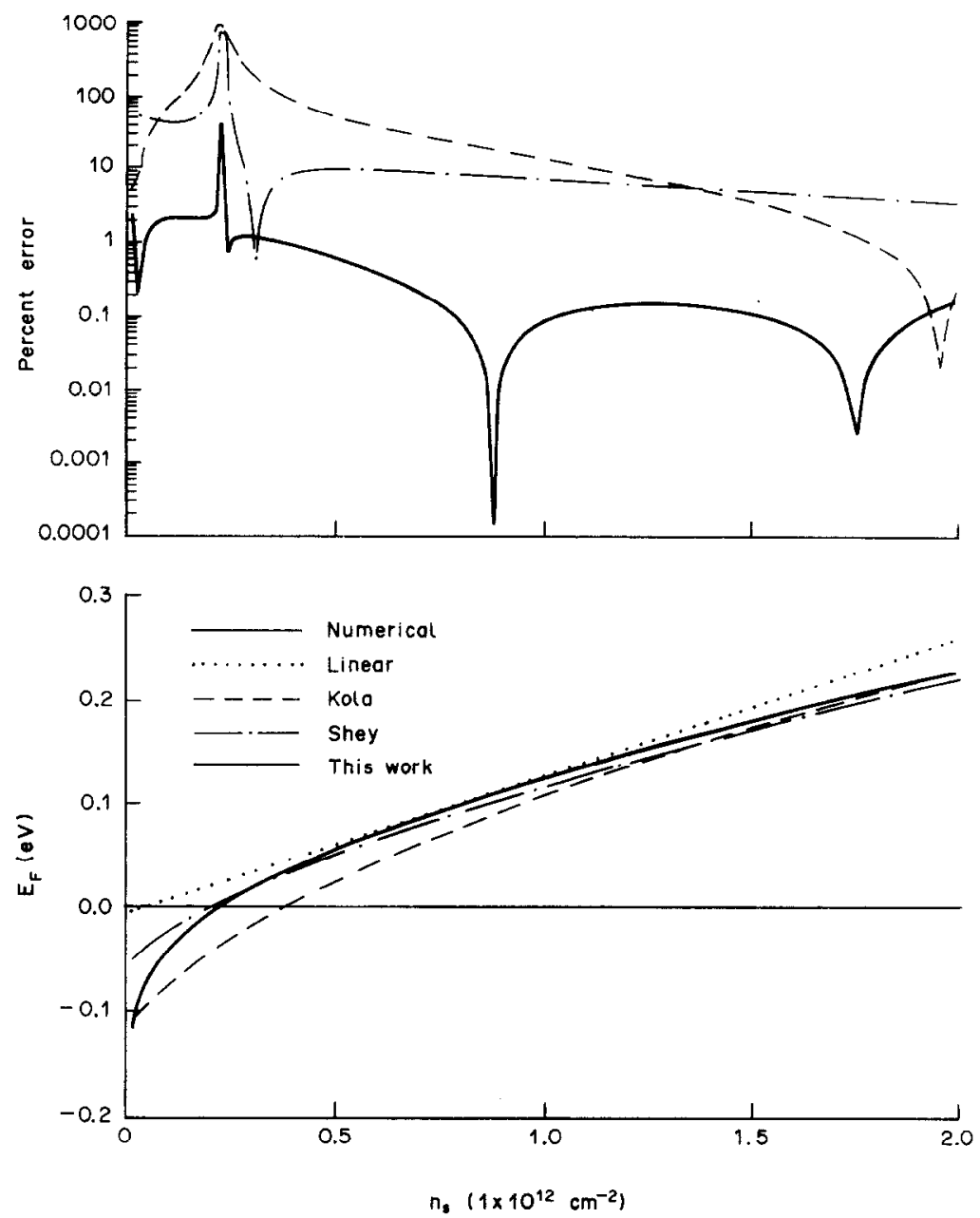

Fig. 1. The lower half of the figure illustrates the exact relation (NUMERICAL) between the Fermi level and sheet carrier density in a two level triangular quantum well and various approximate representations.

The upper half of the figure illustrates the absolute per cent error of the nonlinear representations.

limited by the assumptions that the AlGaAs composition is $30 \%$ AlAs and that the background acceptor concentration in the GaAs is $3 \times 10^{14} / \mathrm{cm}^{3}$. Furthermore, the seminal work from which eqns (10) and (11) are obtained, present results in graphical form from a limited set of parameters. Paasch et al. describe the results of self consistent calculations for $n_{\mathrm{s}}$ as a function of the surface electric field in a GaAs MIS (metal-insulator-semiconductor) structure at $300 \mathrm{~K}[16]$. A quasi-empirical formula is also derived to express the results analytically for arbitrary acceptor concentrations in the GaAs channel. Using the prescribed formula it is straightforward to obtain coefficients appropriate to eqn (12) for an arbitrary acceptor concentration using a least squares fitting routine. Table 2 lists coefficients for several acceptor densities in the GaAs at $300 \mathrm{~K}$. The results for acceptor concentrations of $3 \times 10^{13}, 3 \times 10^{14}$ and $3 \times 10^{15} / \mathrm{cm}^{3}$ are shown graphically in Fig. 2 .

Having derived a polynomial expression for $V_{\mathrm{Fi}}$ the problem of deriving a drain $I-V$ characteristic may be reexamined. As before, the fundamental current equation is $I_{\mathrm{D}}=Z v(x) Q_{\mathrm{s}}(x)=Z q n_{\mathrm{s}}(x) v(x)$. Once again using the two-piece linear approximation $v(x)=\mu\left(\mathrm{d} V_{\mathrm{C}} / \mathrm{d} x\right)$ we expand the derivative as $\mathrm{d} V_{\mathrm{C}} / \mathrm{d} x=\left(\mathrm{d} V_{\mathrm{C}} / \mathrm{d} n_{\mathrm{s}}\right)\left(\mathrm{d} n_{\mathrm{s}} / \mathrm{d} x\right)$. From eqn (5) it follows that

$$
\mathrm{d} V_{\mathrm{C}} / \mathrm{d} n_{\mathrm{s}}=\mathrm{d} V_{\mathrm{Fi}} / \mathrm{d} n_{\mathrm{s}}-d q / \epsilon
$$

and $\mathrm{d} V_{\mathrm{F}} / \mathrm{d} n_{\mathrm{s}}$ is obtained directly from eqn (12).

Table 2. Cofficients of the polynomial represenation of $V_{\mathrm{Fi}}(\mathrm{V})$ at several acceptor concentrations in the GaAs (in $\mathrm{cm}^{-3}$ )

\begin{tabular}{ccccc}
\hline $\mathrm{N}_{\mathrm{a}}$ & $\psi$ & $\phi$ & $\xi$ & $v$ \\
\hline $3 \times 10^{13}$ & -0.2025448 & 0.4833097 & -0.6813648 & 0.2867762 \\
$6 \times 10^{13}$ & -0.2005101 & 0.4698460 & -0.6598437 & 0.2757153 \\
$9 \times 10^{13}$ & -0.2015693 & 0.4674208 & -0.6508956 & 0.2696550 \\
$3 \times 10^{14}$ & -0.2165497 & 0.4881685 & -0.6430109 & 0.2534555 \\
$6 \times 10^{14}$ & -0.2348812 & 0.5240091 & -0.6564162 & 0.2468331 \\
$9 \times 10^{14}$ & -0.2443743 & 0.5426155 & -0.6630660 & 0.2424707 \\
$3 \times 10^{15}$ & -0.2771697 & 0.6125333 & -0.6988349 & 0.2327652
\end{tabular}

The coefficients assume that $n_{\mathrm{s}}$ is in units of $10^{12} \mathrm{~cm}^{-2}$. An equivalent representation of $E_{\mathrm{Fi}}$ in $\mathrm{eV}$ is obtained by reversing the sign of each coefficient. 
Making the appropriate substitutions and rearranging yields

$$
\begin{aligned}
& I_{\mathrm{D}} d x=Z q \mu\left[(\psi-d q / \epsilon) n_{\mathrm{s}}\right. \\
& \left.\quad+(2 / 3) \phi n_{\mathrm{s}}^{2 / 3}+(1 / 3) n_{\mathrm{s}}^{1 / 3}\right] d n_{\mathrm{s}}
\end{aligned}
$$

which is to be integrated over $x^{\prime}=0$ to $x$ and $n_{\mathrm{s}}=n_{\mathrm{s}}(0)$ to $n_{\mathrm{s}}(x)$. Intgrating the right hand side yields a function of $n_{\mathrm{s}}$ which we define as

$$
N\left(n_{\mathrm{s}}\right)=(1 / 2)(\psi-d q / \epsilon) n_{\mathrm{s}}^{2}+(2 / 5) \phi n_{\mathrm{s}}^{5 / 3}+(1 / 4) n_{\mathrm{s}}^{4 / 3}
$$

simplifying the expression for $I_{\mathrm{D}}$ to

$$
I_{\mathrm{D}}=(Z q \mu / x)\left[N\left(n_{\mathrm{s}}(x)\right)-N\left(n_{\mathrm{s}}(0)\right)\right] .
$$

To find $I_{\mathrm{D} \text {, sat }}$ we set $n_{\mathrm{L}}=n_{\mathrm{s}}(L), n_{0}=n_{\mathrm{s}}(0)$ and note that at the onset of saturation $I_{\mathrm{D} \text {, sat }}=Z q v_{\mathrm{s}} n_{\mathrm{L}}=Z q \mu F_{\mathrm{c}} n_{\mathrm{L}}$. Selection of an arbitrary valid value of $I_{\mathrm{D} \text {, sat }}$ or $n_{\mathrm{L}}$ determines $n_{0}$ as one of the six roots of a polynomial in $n_{0}^{1 / 3}$ defined by eqn (16). The appropriate root defines the gate voltage $V_{\mathrm{G}}^{\prime}$ via eqn (5) with $V_{\mathrm{C}}(0)=R_{\mathrm{S}} I_{\mathrm{D}}$, sat which in turn defines $V_{\mathrm{C}}(\mathrm{L})$, again via eqn (5).

The next level of sophistication entails deriving an expression for $I_{\mathrm{D}}$ using eqn (6) to express the velocityfield relation. The derivation proceeds as before with the result

$$
\begin{array}{r}
I=Z q \mu[n(x) N(x)-n(0) N(0)] / \\
\left\{x+\left(v_{\mathrm{s}} / \mu\right)\left[V_{C}(x)-V_{C}(0)\right]\right\}
\end{array}
$$

where $V_{\mathrm{C}}(x)-V_{\mathrm{C}}(0)$ is an explicit function only of $n_{\mathrm{s}}$ as defined by eqns (5) and (12). The explicit notation $N\left(n_{\mathrm{s}}(x)\right)$ has also been simplified to obtain a more compact result. Obtaining a set of $I-V$ characteristics for eqn (17) is more difficult than for eqn (16) because the electron velocity approaches $v_{\mathrm{s}}$ only asymtotically. The criterion for saturation is $\mathrm{d} I_{\mathrm{D}} / \mathrm{d} V_{\mathrm{C}}(L)=0$. To illustrate one method of solution assume that the device being modeled is an ideal normally on transistor. $V_{G}^{\prime}$ may be selected arbitrarily in the range $V_{\text {off }} \leqslant V_{\mathrm{G}}^{\prime} \leqslant 0$. For a fixed $V_{\mathrm{G}}^{\prime}$ an $I_{\mathrm{D}}-V_{\mathrm{C}}(L)$ curve is

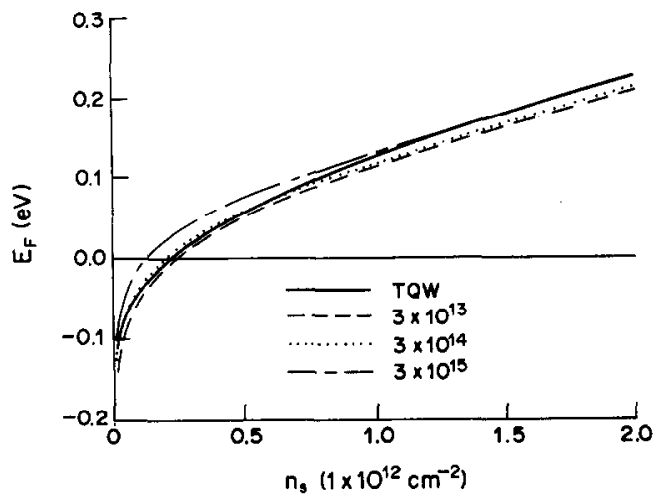

Fig. 2. Interface Fermi level vs sheet carrier density in a triangular quantum well (TQW) and from self consistent solution with various acceptor concentrations (in $\mathrm{cm}^{-3}$ ) in the GaAs. All curves are from the polynomial representation as given in Tables 2 and 3 with a change of sign to obtain $E_{\mathrm{F}}$.

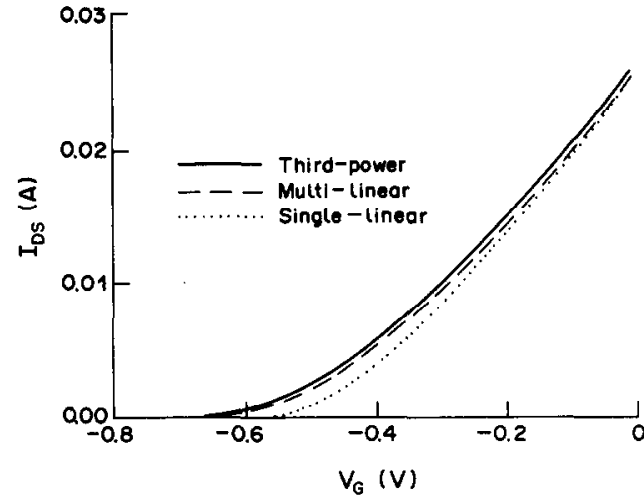

Fig. 3. This figure compares the saturation drain current derived using a two-piece linear velocity-field characteristic and various representations of the function $V_{\mathrm{Fi}}\left(n_{\mathrm{s}}\right)$ at $300 \mathrm{~K}$. The third-power curve is the polynomial representation in terms of $n_{s}^{1 / 3}$.

built up from $I_{\mathrm{D}}=0$. Selecting a small $I_{\mathrm{D}}, n(0)$ is obtained from eqn (5) with $V_{\mathrm{C}}(0) \equiv R_{\mathrm{S}} I_{\mathrm{D}} \cdot n(0)$ is then determined by eqn (17) and $V_{\mathrm{C}}(L)$ by eqn (5) again. $I_{\mathrm{D}}$ is repeatedly incremented until the saturation condition is met. The derivative $\mathrm{d} I_{\mathrm{D}} / \mathrm{d} V_{\mathrm{C}}(L)$ may be evaluated analytically as $\left(\mathrm{d} I_{\mathrm{D}} / \mathrm{d} n_{\mathrm{s}}(L)\right)\left(\mathrm{d} n_{\mathrm{s}}(L) /\right.$ $\left.\mathrm{d} V_{\mathrm{C}}(L)\right)$ but this result is a long and unwieldy polynomial expression in $n_{\mathrm{s}}^{1 / 3}$. To avoid the problem of determining the onset of saturation a composite velocity-field characteristic as suggested by Rohdin and Roblin may be applied[9]. In this approach eqn (6) is adjusted to fit the true velocity-field characteristic up to the peak velocity. Beyond the peak velocity a constant $v_{\mathrm{s}}$ is applied. This approximation reintroduces a well defined $F_{\mathrm{c}}$ which simplifies the determination of $I_{\mathrm{D} \text {, sat }}$ to an explicit procedure similar to that used for eqn (16).

The final feature of the model is the introduction of a low field mobility which is dependent on the channel electron density at $x=0$. As reported by Sakaki et al. the low field mobility in a modulation doped structure is functionally dependent on $n_{\mathrm{s}}$ as $\mu=\mu_{0}\left(n_{\mathrm{s}} n_{\mathrm{s} 0}\right)^{\gamma}$ where $\gamma$ is temperature dependent. Typical values are $1.1,0.5$ and 0.3 at 10,77 and $300 \mathrm{~K}$, respectively[11]. Contrary to the approach of Ref. [11], the present application applies this dependence only to determine a mobility which is constant along the channel in the two-piece linear approximation or modified as prescribed by eqn (6). Modifying a velocity-field characteristic by a position dependent mobility at ficlds greater that about $300 \mathrm{~V} / \mathrm{cm}$ where polar optical phonon emission dominates transport does not seem to be physically justified[17]. A more reasonable approach is obtained by simply replacing $\mu$ in eqns (16) and (17) by $\mu_{0}\left(n_{\mathrm{s}}(0) / n_{\mathrm{s} 0}\right)^{7}$.

To illustrate the effect of the various models a series of comparisons within the triangular well approximation are made. The prototype model is that of Drummond et al.[2] using a two-piece linear velocity-field approximation and a linear $V_{\mathrm{Fi}}\left(n_{\mathrm{s}}\right)$ relation. Figure 3 compares $I_{\mathrm{D} \text {. sat }}$ vs $V_{\mathrm{G}}$ for the single 
linear model, the multilinear model and eqn (16) for a fixed set of material and device parameters as listed in Table 3 (the parameters in Table 3 will be used uniformly throughout the rest of the paper). Each of these models uses the same velocity field relation (with $\gamma=0$ ) and varies only in the description of the channel electron density as a function of $V_{\mathrm{G}}$ and $V_{\mathrm{D}}$. With each added level of sophistication the near threshold currents increase. To explain the increase consider the linear approximation as shown in Fig. 1. For small $n_{\mathrm{s}}, n_{\mathrm{s}}$ varies less rapidly with $V_{\mathrm{F}}$ than the linear approximation. Hence any linear approximation will pinch off the transistor more rapidly than an exact model.

Beyond the triangular well approximation the effect of acceptors in the GaAs is also greatest near threshold. Referring to Fig. 2, at the lower concentrations the rate of change of $E_{\mathrm{F}}$ with $n_{\mathrm{s}}$ is increased for $n_{\mathrm{s}}<5 \times 10^{11} / \mathrm{cm}^{2}$. This translates to an increase in the near threshold current. A plot of the saturated drain current using the two-piece linear velocity-field relation for various acceptor densities in the GaAs is shown in Fig. 4. To illustrate the differences more clearly the current ratio $I_{\mathrm{Na}} / I_{\mathrm{TQw}}$ relating the current obtained with a given acceptor concentration, $N_{\mathrm{a}}$, to that obtained in the approximation of the triangular quantum well is shown in Fig. 5.

A critical issue in device modeling is to obtain agreement between theoretical and experimental device characteristics using known material parameters and independently measured device parameters

Table 3. Heterostructure and device parameters used to generate the transfer characteristics of Figs 3, 4, 6 and 7

\begin{tabular}{lll}
\hline Material & \multicolumn{1}{c}{ Transport } & \multicolumn{1}{c}{ Device } \\
\hline$x=0.33$ & $\mu=0.6 \mathrm{~m}^{2} \mathrm{~V}^{-1} \mathrm{~s}^{\prime}$ & $L=1 \mu \mathrm{m}$ \\
$N_{\mathrm{d}}=1 \times 10^{18} \mathrm{~cm}^{*}$ & $t_{\mathrm{s}}=2 \times 10^{5} \mathrm{~ms}^{\prime}$ & $\mathrm{Z}=300 \mu \mathrm{m}$ \\
$d_{\mathrm{s}}=10 \mathrm{~nm}$ & & $R_{\mathrm{S}}=5 \Omega$ \\
\hline
\end{tabular}

The temperature is $300 \mathrm{~K}$ in all cases and $x$ is the AlAs mol fraction in the $\mathrm{Al}_{*} \mathrm{Ga}_{1}$. As.

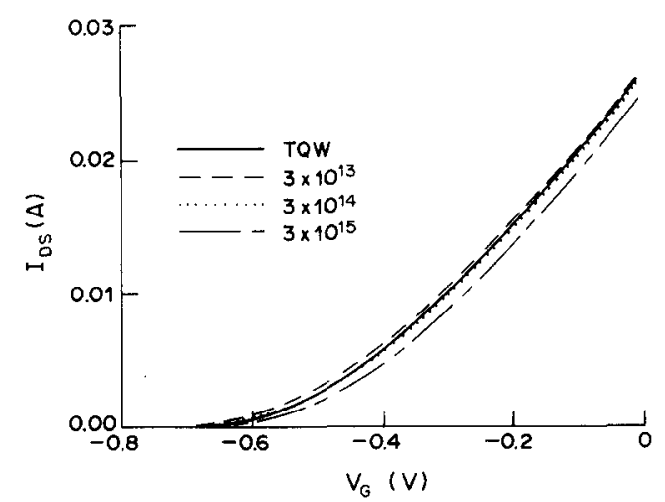

Fig. 4. Saturation drain currents derived using a two-piece linear velocity-field characteristic and various representations of $V_{\mathrm{Fi}}\left(n_{\mathrm{s}}\right)$ at $300 \mathrm{~K}$. In the triangular quantum well approximation there are no acceptors. The other curves show the effect of various acceptor levels (in $\mathrm{cm}^{-3}$ ) as originally derived from a self consistent calculation.

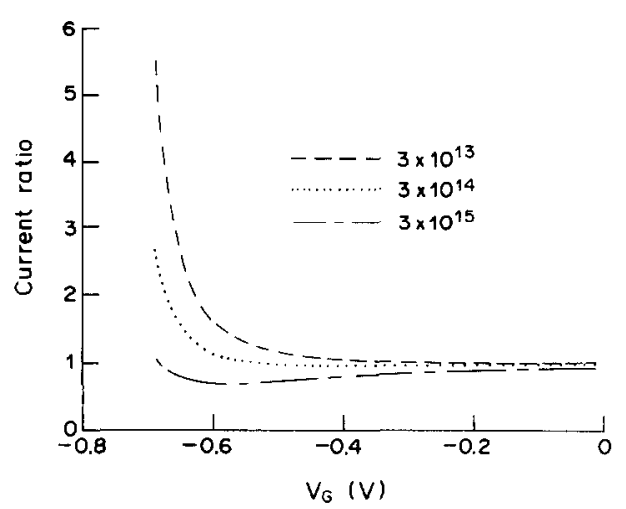

Fig. 5. Current ratios $I_{\mathrm{Na}} / I_{\mathrm{TQW}}$ derived from the results of Fig. 4.

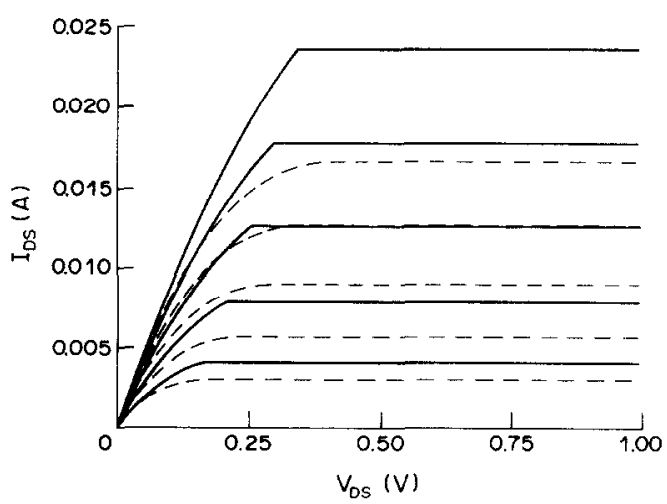

Fig. 6. This figure compares the application of eqns (16) $(-)$ and eqn (17) $(--)$ in the triangular well approximation at $300 \mathrm{~K}$. The gate voltage decreases from $-0.05 \mathrm{~V}$ in steps of $0.1 \mathrm{~V}$.

(i.e. source and drain resistances). Often, more concern is paid to having a smooth transition to saturation than for obtaining an accurate description of device operation in the triode region. To illustrate the effect that the choice of a particular velocity-field characteristic has on device characteristics Fig. 6 compares the results of eqns (16) and (17) with $\gamma=0$ in the triangular well approximation. The two velocity-field characteristics chosen are very nearly extreme representations for this relationship. A dramatic reduction in the saturation current is evident when eqn (6) is used to describe the velocity-field characteristic. Figure 7 shows the effect of using $\gamma=1 / 3$ in eqn (17) again using the triangular well approximation. As the low field mobility decreases the current levels decrease as well. This effect is comparable when applied to eqn (16) with the two piece linear-velocity field characteristic. Figures 6 and 7 illustrate the fact that saturation current levels depend not only on the saturated electron velocity and low field mobility but on how the transition between the two regimes is described.

In the preceeding discussion analytic MODFET models based on improved physical descriptions of the two dimensional electron gas density in the 


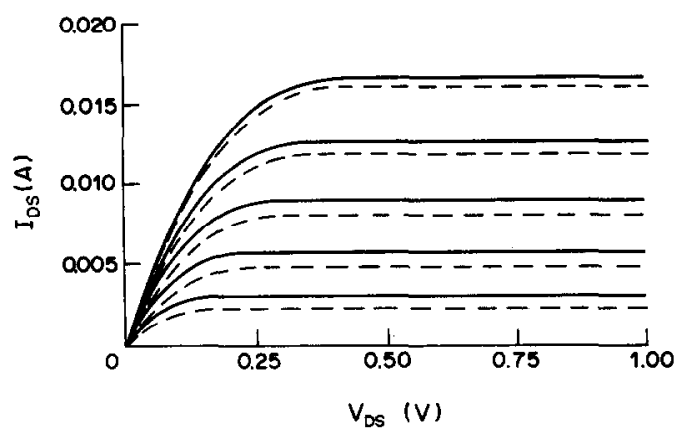

Fig. 7. This figure compares the transfer characteristics obtained from eqn (17) with and without a carrier concentration dependent mobility, $\mu=\mu_{0}\left(n_{\mathrm{s}} / n_{\mathrm{s} 0}\right)^{\prime \prime}$ in the triangular well approximation at $300 \mathrm{~K}$. The gate voltage decreases from $-0.05 \mathrm{~V}$ in steps of $0.1 \mathrm{~V}$. In the first case $y=1 / 3$ $(--)$ and in the latter $\gamma=0(-)$. In this case $\mu_{0}$ corresponds to $\mu$ in Table 3 .

channel have been presented. It is the intent of these models to provide a more physical description of a MODFET in an analytic framework. Presumably, this implies that a theoretical description derived from extrinsic material and device parameters will agree more closely with experimental characteristics than otherwise. This is obviously desirable in computer-aided simulations of MODFET circuits. It is interesting to note that the velocity-field relation is itself a function of the heterojunction parameters as determined by Masselink et al. [18]. The composite approximation offered by Rhodin and Roblin[9] seems well suited to their analytic description as a function of $\mathrm{Al} \mathrm{mol} \mathrm{fraction.} \mathrm{In} \mathrm{conclusion,} \mathrm{the} \mathrm{models}$ presented here are relatively simple, they are analytic, they provide a new and more physical description of MODFET operation and yield new insights as to how they operate.
Acknowledgements - This work was supported in part by the U.S. Army Research Office. The authors would like to thank Dr J. East and Dr S. Tiwari for their insights and advice.

\section{REFERENCES}

1. D. Delagebeaudeuf and N. T. Linh, IEEE Trans. Electron Devices 29, 955 (1982).

2. T. J. Drummond, H. Morkoc, K. Lee and M. S. Shur IEEE Electron Device Lett. 3, 338 (1982).

3. D. Delagebeaudeuf, P. Delescluse, M. Laviron, P. N. Tung, J. Chaplart, J. Chevrier and N. T. Linh, Inst. Phys. Conf., Ser. No. 65, p. 393 (1982).

4. D. C. Tsui, A. C. Gossard, G. Kaminsky and W Wiegmann, Appl. Phys. Lett. 39, 712 (1981).

5. K. Hirakawa and H. Sakaki, Phys. Rev. B 32, 8291 (1986).

6. M. L. Majewski, IEEE Trans. Electron Devices 34, 1902 (1987).

7. C.-S. Chang and H. R. Fetterman, IEEE Trans. Electron Devices 34, 1456 (1987).

8. W. A. Hughes and C. M. Snowden, IEEE Trans. Electron Devices 34, 1617 (1987).

9. H. Rhodin and P. Roblin, IEEE Electron Devices 33, 1617 (1987).

10. C. Z. Cil and S. Tansal, IEEE Electron Devices Lett. 6, 434 (1985).

11. H. Sakaki, J.-I. Motohisa and K. Hirakawa, IEEE Electron Devices Lett. 9, 133 (1988).

12. A. N. Khondker, A. F. M. Anwar, M. A. Islam, L. Limoncelli and D. Wilson, IEEE Electron Devices 33, 1825 (1986).

13. S. Kola, J. M. Golio and G. N. Maracas, IEEE Electron Devices Lett. 9, 136 (1988).

14. M. J. Moloney, F. Ponse and H. Morkoc, IEEE Electron Devices 32, 1675 (1985).

15. A.-J. Shey and W. H. Ku, IEEE Electron Devices Lett. 9, 624 (1988).

16. G. Paasch, K. Tarnay, J.-P. Zollner and H. Ubensee, Physica Status Solidi 97a, K107 (1986).

17. H. P. Lee, D. Vakhshoori, Y. H. Lo and S. Wang, $J$. appl. Phys. 57, 4814 (1985).

18. W. T. Masselink, N. Braslau, W. I. Wang and S. L. Wright, Appl. Phys. Lett. 51, 1533 (1987). 